\title{
Exploration of serum- and cell culture- derived exosomes from dogs
}

\author{
Matias Aguilera-Rojas', Brit Badewien-Rentzsch¹, Johanna Plendl ${ }^{2}$, Barbara Kohn ${ }^{3}$ and Ralf Einspanier ${ }^{1 *}$ (D
}

\begin{abstract}
Background: Exosomes are defined as extracellular membrane vesicles, 30-150 nm in diameter, derived from all types of cells. They originate via endocytosis and then they are released through exocytosis to the extracellular space, being found in various biological fluids as well as in cell culture medium. In the last few years, exosomes have gained considerable scientific interest due to their potential use as biomarkers, especially in the field of cancer research. This report describes a method to isolate, quantify and identify serum- and cell culture-derived exosomes from dog samples, using small volumes $(100 \mu \mathrm{L}$ and $1 \mathrm{~mL}$, respectively).

Results: Quantification and sizing of exosomes contained in serum and cell culture samples were assessed by utilizing nanoparticle tracking analysis, transmission electron microscopy and immunoelectron microscopy. Detected particles showed the normal size $(30-150 \mathrm{~nm})$ and morphology described for exosomes, as well as presence of the transmembrane protein CD63 known as exosomal marker.

Conclusions: Based on a validated rapid isolation procedure of nanoparticles from small volumes of different types of dog samples, a characterization and exploration of intact exosomes, as well as facilitation for their analysis in downstream applications was introduced.
\end{abstract}

Keywords: Exosomes, Serum, Cell culture medium, Dog, Transmission electron microscopy, Nanoparticle tracking analysis, Biomarkers

\section{Background}

Exosomes are extracellular nano-sized membrane vesicles, reported as $30-150 \mathrm{~nm}$ in diameter, derived from all types of cells and released into practically all biological fluids such as blood, urine, cerebrospinal fluid, milk, sputum, saliva, seminal fluid, as well as into cell culture medium $[1,2]$. These vesicles originate via endocytosis, initially forming endosomes and followed by invagination of the endosomal membrane to create multivesicular bodies (MVBs). Afterwards through exocytosis, the content of the MVBs is released as exosomes to the extracellular space once merging with the plasma membrane $[3,4]$.

The exosomal membrane consists mostly of lipids and proteins, while the luminal cargo is mainly represented by proteins and nucleic acids, including mRNAs, microRNAs, other non-coding RNAs and DNA [5-7]. Exosomes have been proven to possess several functions, for instance,

\footnotetext{
* Correspondence: Ralf.Einspanier@fu-berlin.de

${ }^{1}$ Institute of Veterinary Biochemistry, Department of Veterinary Medicine,

Freie Universität Berlin, 14163 Berlin, Germany

Full list of author information is available at the end of the article
}

intercellular communication, genetic exchange and antigen presentation, allowing cells to transport their cargo in a short and long distance manner and subsequently having a significant effect at a cellular and biological level $[6,7]$. Since exosomes are of endosomal origin, they contain a distinct set of proteins involved in membrane transport and fusion (e.g. Rab GTPases, annexins, flotillin), biogenesis of MVBs (Alix, TSG101), major histocompatibility complex class I and II, in processes requiring heat shock proteins (hsc70 and 90), integrins and tetraspanins (e.g. CD63, CD9, CD81 and CD82) $[6,8,9]$. Even though some of these proteins are used as exosome markers, exosomal protein composition might differ based on the origin of the cells or tissue $[7,10]$.

Analyses of cargo proteins and nucleic acids present in exosomes show significant potential to be employed as exosomal biomarkers. Taking this into consideration, together with the ability to easily isolate exosomes from body fluids (liquid biopsy), these vesicles may deliver an additional valuable non-invasive biomarker for predisposition, prognosis and treatment monitoring in the cancer research field 
[7, 11]. Furthermore, when understanding endogenous transmission of distinct macromolecules between tissues via exosomes, a (dys) functional cell-cell communication could be focused (diagnostic tool) and subsequently modified (therapeutic tool).

In this report we describe a method to isolate and identify serum- and cell culture-derived exosomes from dog samples. This study provides comprehensive techniques such as transmission electron microscopy, nanoparticle tracking analysis and immunodetection to identify and characterize exosomes, allowing them to be quantified and sized, as well as characterized through specific morphology and a distinct protein expression.

\section{Methods}

\section{Blood serum}

Samples $(n=10)$ were gathered from 5 female and 5 male dogs of different ages (between 1 and 7 years old), non-cancer $(n=6)$ and cancer patients $(n=4)$, presented at the Small Animal Clinic, Department of Veterinary Medicine at the Freie Universität Berlin. Blood samples were collected in tubes without anticoagulant and left at room temperature to allow clotting for $30 \mathrm{~min}$ to $2 \mathrm{~h}$. The main portion of the serum was used for the original diagnostic laboratory analyses, while the remaining amount was employed for this study. The protocol to separate and store serum was based on a published technical note from QIAGEN (miRNeasy Serum/Plasma Handbook 02/2012). Briefly, tubes were first centrifuged at $2000 \mathrm{x}$ g for $10 \mathrm{~min}$ at $4{ }^{\circ} \mathrm{C}$ to separate residual cellular components of the blood. The supernatant was then placed in another tube and centrifuged at $16,000 \mathrm{x}$ g for $10 \mathrm{~min}$ at $4{ }^{\circ} \mathrm{C}$ to separate any left cellular debris. Afterwards, the purified serum was taken and stored in $-80^{\circ} \mathrm{C}$ until exosome isolation.

\section{Cell cultures \\ C2 cell line}

C2 cells, a canine mast cell tumour cell line, were kindly provided in August 2016 by Dr. Patrice Dubreuil (Centre de Recherche en Cancérologie de Marseille, Inserm U1068, Marseille, France), after previous consent of the cell line originator, Dr. Warren Gold (University of California San Francisco, School of Medicine, California, USA) [12]. Cells were cultured in RPMI 1640 medium, supplemented with $10 \%$ foetal bovine serum (FBS) superior, $100 \mathrm{U} / \mathrm{mL}$ penicillin/streptomycin (all from Biochrom, Berlin, Germany), $1 \mathrm{mM} / \mathrm{mL}$ sodium pyruvate and $2 \mathrm{mM} / \mathrm{mL}$ glutamine (both from Sigma, MO, USA), and incubated in a $5 \% \mathrm{CO}_{2}$ atmosphere at $37{ }^{\circ} \mathrm{C}$. Special culture conditions were applied before exosomes were harvested (see exosome isolation paragraph).

\section{Primary canine fibroblasts culture}

Fibroblasts (FBs) were obtained from a portion of healthy skin of a female Golden Retriever, within $20 \mathrm{~min}$ after the animal was euthanized at the Small Animal Clinic, Department of Veterinary Medicine at the Freie Universität Berlin. The skin was collected in sterile Dulbecco's phosphate buffer saline (DPBS) (Sigma, MO, USA) and then placed in a Petri dish. Dermis was separated from epidermis using sterile forceps and scalpels. The dermis was cut in small pieces $(1 \times 3 \mathrm{~mm}$ approx. $)$ and washed in DPBS, supplemented with $100 \mathrm{U} / \mathrm{mL}$ penicillin/streptomycin and $250 \mu \mathrm{g} / \mathrm{mL}$ amphotericin B (Biochrom, Berlin, Germany). Then, a 5 min centrifugation at $300 \mathrm{x} g$ was performed and the supernatant was discarded. The sediment, representing the FBs, was resuspended in an enzymatic digestion medium containing 0.15\% collagenase I (Biochrom, Berlin, Germany), RPMI 1640 medium, supplemented with antibiotic and fungicide as described above, and $1 \% 70 \mathrm{mM} \mathrm{CaCl}_{2}$ (Merck, Darmstadt, Germany). The sample was transferred into a Petri dish and incubated at $37^{\circ} \mathrm{C}$ for $2 \mathrm{~h}$ under constant agitation, then placed into a sterile $50 \mathrm{~mL}$ tube and centrifuged $5 \mathrm{~min}$ at $300 \mathrm{x} \mathrm{g}$, the supernatant was discarded. The pellet was washed twice in warm $\left(37{ }^{\circ} \mathrm{C}\right)$ RPMI 1640 medium, supplemented with 20\% FBS, $100 \mathrm{U} / \mathrm{mL}$ penicillin/streptomycin, $250 \mu \mathrm{g} / \mathrm{mL}$ amphotericin $\mathrm{B}, 1 \mathrm{mM} / \mathrm{mL}$ sodium pyruvate and $2 \mathrm{mM} / \mathrm{mL}$ glutamine, and centrifuged $5 \mathrm{~min}$ at $300 \mathrm{x} \mathrm{g}$. Lastly, the resulting pellet was seeded in a T25 flask in $7 \mathrm{ml}$ of the same medium used for the last two washing steps and incubated in a $5 \% \mathrm{CO}_{2}$ atmosphere at $37{ }^{\circ} \mathrm{C}$. The first passage was performed 10 days after seeding and passage number 5 was used for the exosome isolation. Special culture conditions were applied before exosomes were harvested (see exosome isolation paragraph).

\section{Exosome isolation \\ Serum samples}

Exosome isolation from serum samples was accomplished using a commercial kit (Total Exosome Isolation Reagent from serum; Invitrogen, Vilnius, Lithuania) following the manufacturer's protocol. Briefly, purified serum was passed through a $0.22 \mu \mathrm{m}$ pore PVDF filter (Rotilabo, Karlsruhe, Germany). After that, $100 \mu \mathrm{L}$ of filtered serum was mixed with $20 \mu \mathrm{L}$ of reagent and incubated at $4{ }^{\circ} \mathrm{C}$ for $30 \mathrm{~min}$. Then, samples were centrifuged at $16,000 \mathrm{x}$ g for $10 \mathrm{~min}$ at room temperature and the supernatant was discarded. The pellet containing exosomes was resuspended in 20 to $50 \mu \mathrm{L}$ of DPBS, depending on the downstream applications.

\section{Cell culture samples}

In cell culture medium from the $\mathrm{C} 2$ cell line and from the primary FBs culture, exosome isolation was performed utilizing a commercial kit (Total Exosome 
Isolation Reagent - from cell culture media; Invitrogen, Vilnius, Lithuania), although some modifications to the manufacturer's protocol were applied. For this purpose, prior to culturing cells for exosome isolation, $50-80 \%$ confluent $\mathrm{C} 2$ cells and primary FBs were washed twice in DPBS and further cultured in an exosome-free medium as described above, except for using exosome-depleted FBS (Gibco, USA). Briefly, cell culture medium was harvested after 48 and $72 \mathrm{~h}$ of incubation with exosome-depleted medium and centrifuged at room temperature; first, $5 \mathrm{~min}$ at $300 \mathrm{x}$ g to remove floating cells and a subsequent $30 \mathrm{~min} 3000 \mathrm{x}$ g centrifugation to eliminate cellular debris. Afterwards, the purified medium was passed through a $0.22 \mu \mathrm{m}$ pore PVDF filter and then $1 \mathrm{~mL}$ of filtered medium was mixed with the volume of reagent indicated by the manufacturer. The mixture was incubated at $4{ }^{\circ} \mathrm{C}$ overnight and finally centrifuged at $4{ }^{\circ} \mathrm{C}$ at $11,000 \mathrm{x}$ g for $60 \mathrm{~min}$. The pellet containing exosomes was re-suspended in 20 to $50 \mu \mathrm{L}$ of DPBS, depending on the downstream applications.

\section{Transmission electron microscopy (TEM)}

To identify exosomes and investigate their ultrastructural morphology, a Zeiss EM 109 transmission electron microscope (Carl Zeiss, Oberkochen, Germany) operating at $80 \mathrm{kV}$ was utilized, following the protocol developed by Théry et al. (2006) [13], with some modifications.

\section{Native exosomes}

For analysis of native exosomes, PBS-suspensions containing unfixed exosomes were differentially diluted in filtered PBS $(0.22 \mu \mathrm{m}$ pore PVDF filter). For serum-derived exosomes; undiluted suspension and 1:100, 1:1000 and 1:2000 dilutions were used, while for culture-derived exosomes; undiluted suspension and 1:50, 1:100 and 1:200 dilutions were applied. Formvar-carbon-coated 100 mesh nickel grids (Plano, Wetzlar, Germany) were laid on a $5 \mu \mathrm{L}$ drop of the exosome-suspension and incubated $20 \mathrm{~min}$ at room temperature for adhesion (coated side of the grid facing the suspension), then washed 3 times for $3 \mathrm{~min}$ in filtered PBS. Next, grids were placed 2 times on drops of $50 \mathrm{mM}$ glycine/PBS for $3 \mathrm{~min}$ and then transferred to a drop of $0.5 \%$ bovine serum albumin (BSA)/PBS blocking solution for $10 \mathrm{~min}$. For contrasting the exosomes, grids were laid on $2 \%$ uranyl acetate drops for $6 \mathrm{~min}$, followed by 2 washes with distilled water. Grids were allowed to dry overnight.

\section{Immuno-gold labelled exosomes}

For examination of immuno-gold labelled unfixed exosomes, anti-CD63 (ABIN1440014, antibodies-online), a goat polyclonal multi-species primary antibody, and a secondary antibody anti-goat IgG (whole molecule) labelled with $10 \mathrm{~nm}$ gold (Sigma, MO, USA) were used. The first part of the protocol was identical to the procedure for analysis of native exosomes up to placing the grids on drops of $0.5 \%$ BSA/PBS blocking solution for $10 \mathrm{~min}$. This was followed by an incubation step for $2 \mathrm{~h}$ at room temperature with the primary antibody anti-CD63 (dilution $1: 50$ in $0.5 \% \mathrm{BSA} / \mathrm{PBS}$ ). Afterwards, grids were washed 5 times for $3 \mathrm{~min}$ in drops of $0.5 \% \mathrm{BSA} / \mathrm{PBS}$ and an additional incubation with the secondary antibody anti-goat IgG-10 nm gold (dilution 1:50 in 0.5\% BSA/PBS) was performed for $1 \mathrm{~h}$ at room temperature. Grids were washed again 5 times for $3 \mathrm{~min}$ in $0.5 \%$ BSA/PBS drops, and then laid on $2 \%$ uranyl acetate drops for 6 min for contrasting, followed by 2 washes in distilled water. Grids were allowed to dry overnight.

\section{Nanoparticle tracking analysis (NTA)}

Quantification and size determination of dog exosomes purified from serum and cell culture medium was assessed by using the NanoSight NS500 instrument (Malvern, Worcestershire, UK). The NTA 3.0 (build 0064) software visualizes and analyses nanoparticles in real time by associating Brownian motion with particle size. Fresh serum- and cell culture-derived exosomes samples were processed in duplicate and diluted in filtered PBS $(0.22 \mu \mathrm{m}$ pore PVDF filter $)$ until reaching a concentration between 10 and 100 particles per image (optimal $\sim 50$ particles per image) before examination with the NTA system [14]. The instrument was set up to operate at $25{ }^{\circ} \mathrm{C}$, three videos, $30 \mathrm{~s}$ each, were recorded for each specimen and outcomes were analysed with the NTA software.

\section{Statistical analysis}

The data analysis was performed using the software Microsoft Excel 2010 (Microsoft, Redmond, WA, USA), through one-way analysis of variance (ANOVA) and Bonferroni corrected post-hoc Student's t-tests. $P$ value $<0.05$ was considered as significant.

\section{Results}

A rapid protocol was validated to isolate nanoparticles from dog samples suitable to further detect size, quantity and evaluate selected protein expression.

\section{Size and quantification of exosomes by NTA}

A suitable real-time visualization and analysis of exosomes present in fluid samples could be easily performed by the NTA system, both in blood serum (Table 1, Fig. 1a) and in culture media (Table 2, Fig. 1b and c).

\section{Serum samples}

$100 \mu \mathrm{L}$ of canine serum was employed to isolate serum-derived exosomes from 6 non-cancer and 4 cancer dog patients. Most of the observed nanoparticles 
Table 1 Exosome concentration and size distribution

\begin{tabular}{llll}
\hline Sample ID & Exosome concentration $(x E 10 / \mathrm{mL})$ & Particle size mean $(\mathrm{nm})$ & Type of sample \\
\hline S1 & $403.2+/-25.8$ & $71.3+/-3.1$ & Non-cancer \\
S2 & $107.4+/-6.8$ & $90.5+/-14.5$ & Non-cancer \\
S3 & $322.8+/-24.0$ & $89.5+/-1.4$ & Non-cancer \\
S10 & $198.0+/-19.9$ & $89.9+/-13.2$ & Non-cancer \\
SHB & $374.4+/-21.8$ & $112.5+/-12.8$ & Non-cancer \\
SNT & $219.6+/-17.3$ & $111.3+/-10.7$ & Non-cancer \\
S8 & $397.2+/-18.6$ & $113.9+/-9.0$ & Splenic mast cell tumour \\
SP & $225.6+/-10.4$ & $99.0+/-8.4$ & Prostatic carcinoma \\
S15 & $500.4+/-76.4$ & $84.7+/-1.5$ & Perianal adenoma \\
SVT & $277.2+/-11.3$ & $84.5+/-0.6$ & Vaginal leiomyosarcoma
\end{tabular}

Serum-derived exosomes from non-cancer and cancer dog patients (mean $+/-$ standard error)

were found to be 30 to $150 \mathrm{~nm}$ in diameter (Table 1, Fig. 1a), i.e. the normal size described for exosomes [1, 2], however few particles showed a larger size. The mean size range for serum-derived exosomes observed was between $71.3+/-3.1$ and $113.9+/-9.0 \mathrm{~nm}$. In terms of exosome concentration, in non-cancer patients nanoparticle concentration $(\mathrm{xE} 10 / \mathrm{mL})$ was between $107.4+/-6.8$ and $403.2+/-25.8$, while in cancer patients the lowest and the highest concentrations $(\mathrm{xE} 10 / \mathrm{mL})$ were $225.6+/-10.4$ and 500.4 +/ -76.4 , respectively. Nevertheless, no significant difference $(P>0.05)$ between non-cancer and cancer samples could be calculated, although a large variation within individual samples was detected.

\section{Cell culture medium samples}

Exosomes could be isolated from $1 \mathrm{~mL}$ of culture medium obtained from cultured C2 cells and primary FBs, after 48 and $72 \mathrm{~h}$ of incubation under exosome-depleted media conditions. Likewise in the analysis of dog serum samples, the commercial kit was found suitable for isolating exosomes derived from dog cell cultures. The majority of the nanoparticles exhibited the normal size described for exosomes, 30 to $150 \mathrm{~nm}$ in diameter (Table 2, Fig. 1b and c) $[1,2]$, while a small number was found to be in the 150 $300 \mathrm{~nm}$ range. The mean size range for culture-derived exosomes observed in both types of cultures and both time-points was between $110.3+/-5.0$ and $129.0+/-$ $7.4 \mathrm{~nm}$. In terms of nanoparticle quantification, after 48 and $72 \mathrm{~h} \mathrm{C} 2$ cells cultures showed a significant $(P<0.05)$ $2-3$-fold higher exosome concentration compared to primary FBs cultures but no difference $(P>0.05)$ between incubation times ( 48 vs. $72 \mathrm{~h}$ ) was observed in either group.

\section{Negative controls}

To screen for potentially contaminating particles, samples from all solutions used (PBS, RPMI 1640 medium, RPMI 1640 + exosome isolation kit, and complete culture medium + exosome isolation kit) were analysed as negative controls. The number of particles detected in these fluids was low and did not affect the total concentration of exosomes per mL counted by the NTA system. Therefore, exogenous contamination interacting with a
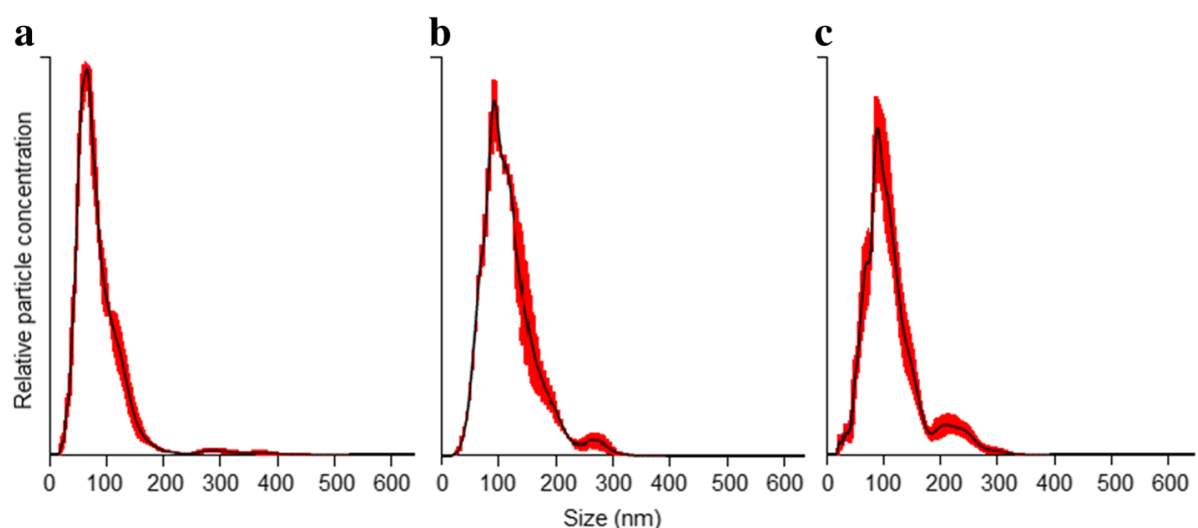

Fig. 1 Nano track analysis size distribution of exosomes isolated from samples of canine origin. a blood serum-derived exosomes, b C2 cell line culture-derived exosomes and (c) primary fibroblasts culture-derived exosomes. Red error bars indicate $+/-$ standard error of the mean 
Table 2 Concentration and size distribution of exosomes

\begin{tabular}{llll}
\hline Sample ID & Exosome concentration $(x E 10 / \mathrm{mL})$ & Particle size mean $(\mathrm{nm})$ & Type of sample \\
\hline C248 & $17.5+/-0.9$ & $120.9+/-2.1$ & C2 cells culture medium, $48 \mathrm{~h}$ incubation \\
C272 & $12.8+/-1.7$ & $118.0+/-4.5$ & C2 cells culture medium, $72 \mathrm{~h}$ incubation \\
FB48 & $6.4+/-0.7$ & $110.3+/-5.0$ & Primary FBs culture medium, $48 \mathrm{~h}$ incubation \\
FB72 & $7.3+/-1.5$ & $129.0+/-7.4$ & Primary FBs culture medium, $72 \mathrm{~h}$ incubation
\end{tabular}

Culture medium-derived exosomes from C2 cell line and primary fibroblasts, after 48 and $72 \mathrm{~h}$ of incubation under exosome-free media conditions (mean $+/-$ standard error)

valid characterization of canine exosomes can be excluded as a factor in our system (Table 3).

\section{Exosome morphology by TEM}

General morphology and ultrastructure of serum- and culture-derived exosomes of canine origin was assessed by using TEM technology, allowing visualization of the characteristic central depression or "cup shape" of exosomes $[15,16]$, either single (Fig. $2 \mathrm{a}$ and b) or aggregated (Fig. 2c and d). All samples revealed single and aggregated nanoparticles; non-diluted samples showed a higher number of exosome aggregates, whereas samples diluted 1:2000 displayed more individual exosomes, yet it was more difficult to localize them on the grids. Morphology and size of the depicted nanoparticles correspond to their exosomal origin, as described in several studies performed in samples of human fluids and cell culture origin [1, 13-15].

\section{Protein expression by immunoelectron microscopy}

Results presented in Fig. 3 revealed the presence of the transmembrane protein CD63 in all samples investigated in this study. It is important to note that not every single exosome observed by TEM expressed this protein. Indeed, the number of exosomes negative for CD63 was slightly greater to the number of exosomes expressing the protein.

\section{Discussion}

\section{Size and quantification}

The commercial kit used states a simple and quick precipitation method for isolation of intact exosomes, allowing them to be collected by a short, low-speed centrifugation easily applicable in most clinical laboratories [2]. The introduced NTA technology overcomes some limitations inherent to TEM-based methods, such as lack of absolute quantification and quick size determination of exosomes, as well as time-consuming protocols for sample preparation. For that reason, NTA-based procedures appear highly suitable to rapidly characterize size distribution and number of exosomes. However, the NTA system is not able to distinguish between extracellular vesicles (EVs) and other similar sized particles, such as clusters of exosomes, cellular debris or protein aggregates. Moreover, especially when working with precipitation methods, co-isolation of non-exosomal particles, for instance larger serum/plasma protein aggregates or lipoproteins, cannot be excluded [1, 17, 18]. These data might explain why we were able to also observe minor signals showing particles between 150 and $400 \mathrm{~nm}$ in the size distribution graph (Fig. 1) in addition to the major peak around $100 \mathrm{~nm}$.

In our approach, culture-derived exosomes were found to have a significantly larger average size than serum-derived specimens, but an obvious size variation between both types of cell cultures was not found. Different studies have provided evidence that EVs vary in size depending on their cells of origin and there are even data published showing variation based on the method of visualization [19-21].

The exosome quantification variability between both cell culture types under the same culture conditions might be explained by the fact that $\mathrm{C} 2$ cells represent a cancer cell line. Since exosome secretion is normally increased in cancer [22], a higher exosome concentration in $\mathrm{C} 2$ cell medium was expected. Compared to primary FBs, the faster growth rate of $\mathrm{C} 2$ cells determines the number of cells contained in each culture flask, an aspect that certainly influences the exosome production. Moreover, it has been well documented that some types of cancer cell lines shed higher amounts of exosomes

Table 3 Negative controls

\begin{tabular}{llll}
\hline Sample ID & Exosome concentration $(x E 7 / \mathrm{mL})$ & Particle size mean $(\mathrm{nm})$ & Type of sample \\
\hline CN1 & $0.88+/-0.36$ & $188.2+/-62.1$ & PBS \\
CN2 & $1.33+/-0.75$ & $138.9+/-69.6$ & RPMl 1640 \\
CN3 & $1.40+/-0.34$ & $130.4+/-11.5$ & RPMl 1640 + Exosome isolation kit \\
CN4 & $1.26+/-0.61$ & $147.6+/-22.6$ & Complete culture medium + Exosome isolation kit
\end{tabular}

Solutions employed during harvest and dilution processing of exosomes (mean $+/-$ standard error) 


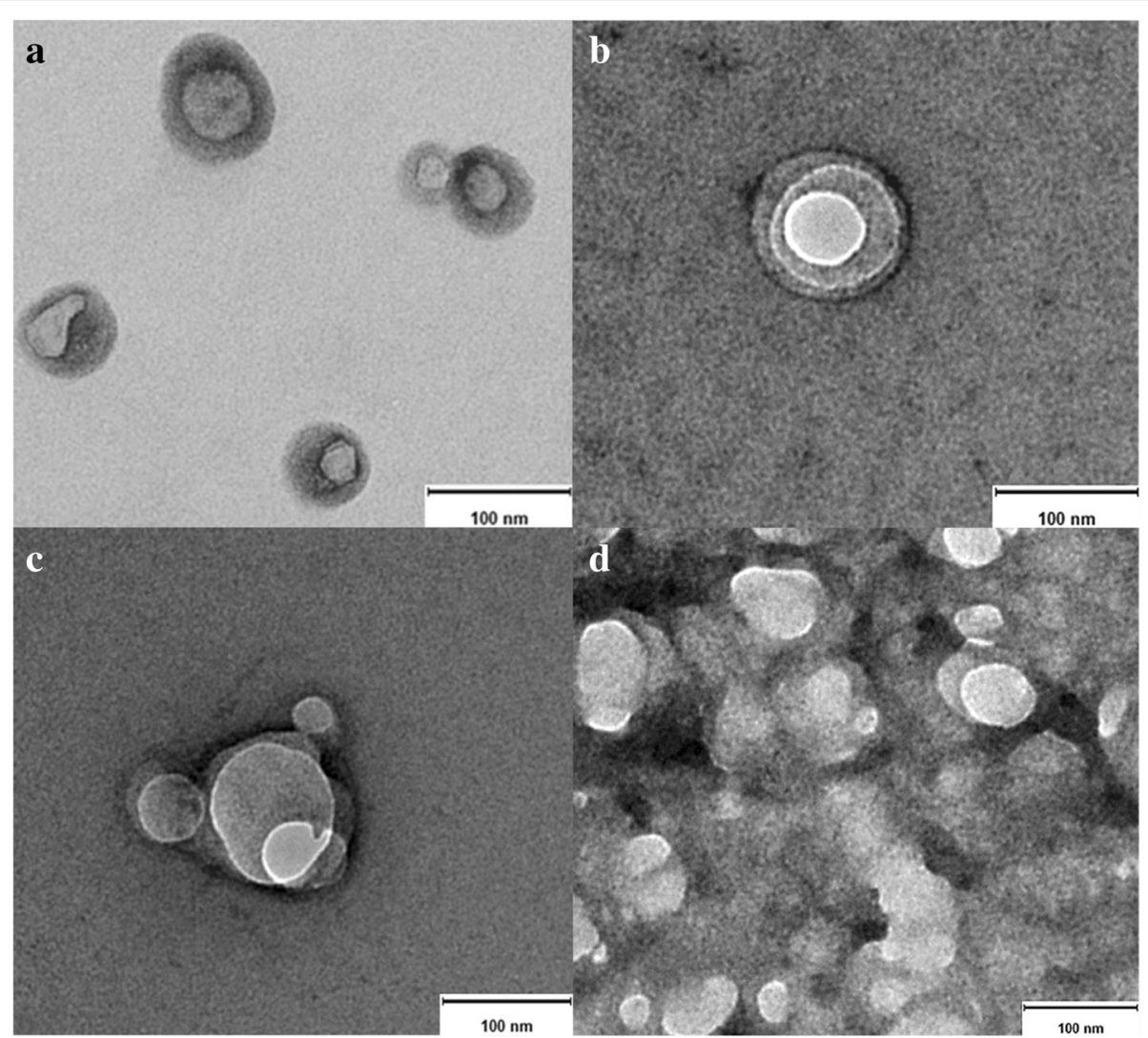

Fig. 2 Transmission electron microscopy of native exosomes isolated from samples of canine origin. a blood serum-derived exosomes, $\mathbf{b}$ C2 cell line culture-derived exosomes and (c and $\mathbf{d}$ ) primary fibroblasts culture-derived exosomes. Size bar $=100 \mathrm{~nm}$

than others and conditions like hypoxia may increase exosome production up to $90 \%$ [23, 24]. Many other elements can also affect exosome shedding in normal and diseased cells, including chemical factors like, calcium, calcium ionophores, phosphatidylinositol 3-kinase, and $\mathrm{pH}$, as well as physical factors such as heat, ischemia, cellular stresses, and loss of cellular attachment [25].

Compared to serum samples, the number of exosomes found in cell culture media was significantly lower. It is known that in vivo exosomes are shed by all types of cells, in normal and diseased conditions [1, 2]. Taking that into consideration, along with the intercellular cross-talks occurring in complex organisms, the total number of cells in a living organism (a dog in this case) releasing exosomes into all body-fluid compartments, is in fact not comparable to the limited number of cells $\left(2-3 \times 10^{6}\right)$ contained in our in vitro culture system.

It is worth to mention that although an aim of this report was to isolate and identify exosomes from different dog serum samples, no differences in size distribution and quantification between non-cancer and cancer dog patients were noticed. Hence, further investigations exploring potential variations between healthy and diseased groups including a larger number of individuals shall follow, since some reports have already shown that cancer cells secrete more exosomes than non-cancer cells [22].

\section{Morphology and protein expression}

Electron microscopy allowed the assessment of morphology and protein expression. Since most optical methods using light scattering to analyse substances or matter, such as flow cytometry and optical microscopy, are hardly able to detect particles smaller than $200 \mathrm{~nm}$, TEM is essential to study the morphology of exosomes and is considered the standard method in this regard $[15,26]$. When referring to morphology of nanoparticles, it involves their overall shape, while TEM detects also ultrastructural differences in their shape, contrast and surface patterns [3]. Although we and other researchers described the morphology of exosomes as cup shaped when observed by TEM, it seems to be an artefact generated by fixation and/or contrasting steps $[13,15]$, that is also associated with shrinking of vesicles [27, 28]. Studies employing scanner electron microscopy and cryo-electron microscopy revealed that exosomes have indeed a round/spherical shape [15, 20, 29, 30].

Immunoelectron microscopy allowed the detection and direct imaging of the transmembrane protein CD63, 


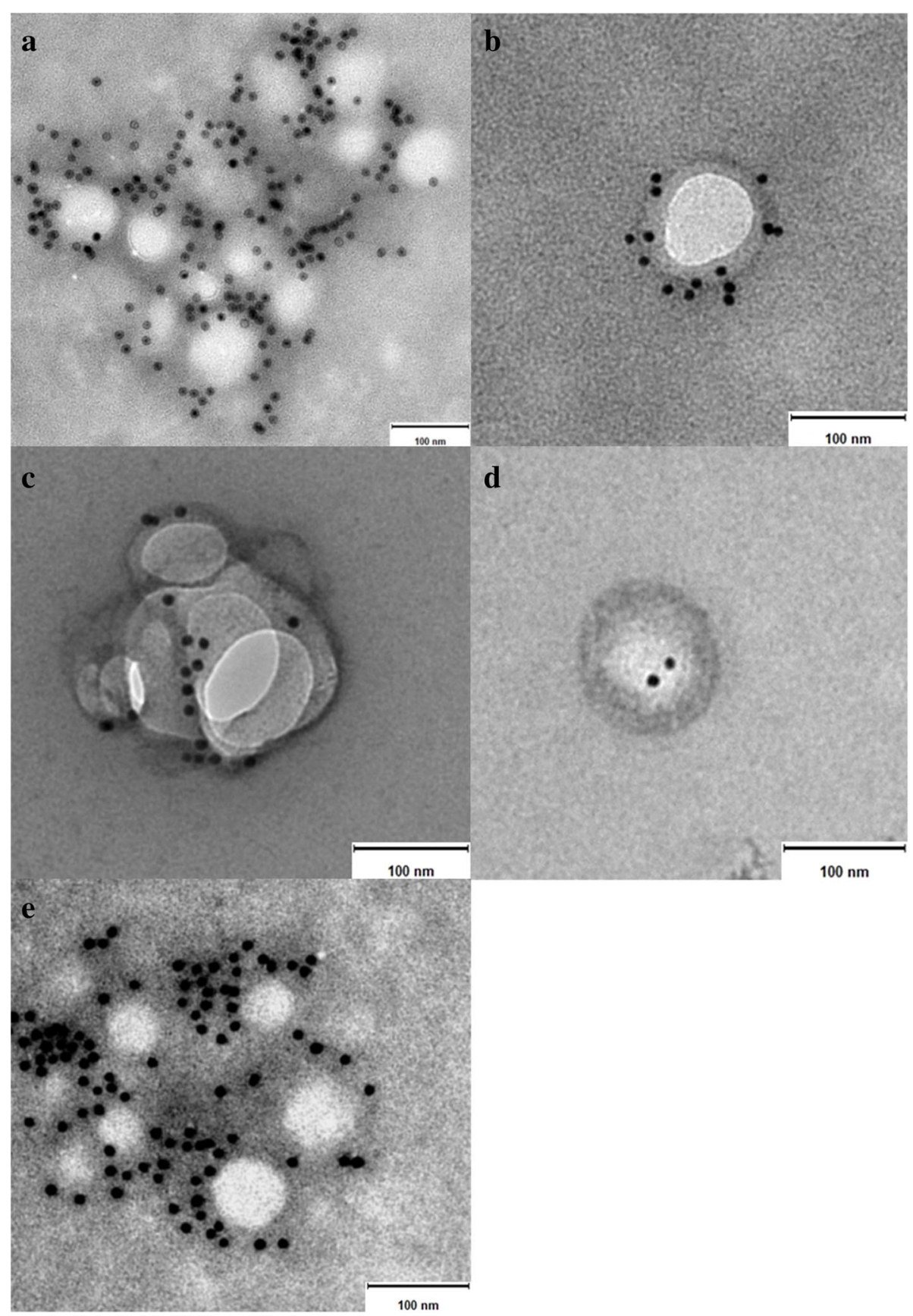

Fig. 3 Immunoelectron microscopy images of exosomes isolated from samples of canine origin. a and $\mathbf{b}$ serum-derived exosomes, c C2 cell line culture-derived exosomes, $\mathbf{d}$ and $\mathbf{e}$ primary fibroblasts culture-derived exosomes. Note the gold particles bound to the exosome membrane indicating presence of the tetraspanin CD63. Size bar $=100 \mathrm{~nm}$

which bound to a selective secondary antibody labelled with gold particles [31] (Fig. 3). Exosomes represent a heterogeneous population of EVs expressing diverse patterns of molecules. Numerous studies have shown that some of these molecules are found frequently in exosomes, and therefore, they have gained support to be used as exosomal markers, e.g. proteins [13, 15, 31]. Since they all bear an endosomal origin, it is expected that exosomes contain different cargos of tetraspanin proteins, a family of membrane proteins. The tetraspanin CD63 is currently being used widely as a molecular exosome marker by diverse research studies in this field $[7,10]$. The tetraspanin family includes a large amount of transmembrane proteins and only the most common members are made available as molecular exosomal markers, including CD63, CD9, CD81 and CD82 [5, 7, 
10]. Several investigations have already demonstrated that the molecular characteristics vary broadly among exosomes from different sources, even across exosomes secreted by the same type of cells [10, 22, 25]. Accordingly, the fact that not all of the observed exosomes expressed CD63 was indeed contemplated.

\section{Conclusion}

Our results evidence the feasibility to easily and rapidly isolate intact exosomes from small volumes of serum, as well as from a tumour cell line and a primary fibroblast culture, all from dog origin, allowing nanoparticles to be analysed in downstream applications. The NTA system provides a quick and easy way to size and quantify exosomes, while TEM facilitates the morphology assessment and distinct immunodetection. The exosome research field has in the past years become an emerging area among researchers of all biological sciences. However, in veterinary medicine it is not yet a well-developed matter. Hence, by demonstrating techniques of isolation, characterization and exploration, this report supports the data until now available in the veterinary diagnostic field, encouraging scientists and clinicians to further explore exosomes of canine origin.

\section{Abbreviations}

ANOVA: Analysis of variance; BSA: Bovine serum albumin; DPBS: Dulbecco's phosphate buffer saline; EVs: Extracellular vesicles; FBs: Fibroblasts; FBS: Foetal bovine serum; MVBs: Multivesicular bodies; NTA: Nanoparticle tracking analysis; PBS: Phosphate buffer saline; PVDF: Polyvinylidene fluoride; TEM: Transmission electron microscopy

\section{Acknowledgments}

The authors thank Dr. L. Bouchet and Prof. Dr. M. Calderon (Institute of Chemistry and Biochemistry, Department of Biology, Chemistry and Pharmacy, Freie Universität Berlin) for their assistance with the NTA system, Ms. F. Ermisch and Ms. V. Holle (Institute of Veterinary Anatomy, Department of Veterinary Medicine, Freie Universität Berlin) for their assistance with the TEM and Ms. P. Schulze (Institute of Veterinary Biochemistry, Department of Veterinary Medicine, Freie Universität Berlin) for her help provided in laboratory work.

\section{Funding}

M. Aguilera-Rojas is a scholarship holder of the doctoral bilateral agreement DAAD/Becas Chile program, between the German and Chilean governments.

\section{Availability of data and materials}

The datasets generated and/or analysed during the current study are available from the corresponding author on reasonable request.

\section{Authors' contributions}

MAR, prepared the manuscript. MAR, BWR and RE, were responsible for the conception and design of the study. BWR and RE supervised the activity planning and execution. RE, JP and BK revised the manuscript critically. All authors have read and approved the final version of the manuscript.

\section{Ethics approval and consent to participate}

The ethics committee from the Governmental Office for Health and Social Affairs Berlin (LAGeSo Berlin), file number StN 0005/17, do not classify the use of residual blood from diagnostic samples from dogs or cats and the use of euthanized animals as animal experimentation.

\section{Competing interests}

The authors declare that they have no competing interests.

\section{Publisher's Note}

Springer Nature remains neutral with regard to jurisdictional claims in published maps and institutional affiliations.

\section{Author details}

'Institute of Veterinary Biochemistry, Department of Veterinary Medicine, Freie Universität Berlin, 14163 Berlin, Germany. ${ }^{2}$ Institute of Veterinary Anatomy, Department of Veterinary Medicine, Freie Universität Berlin, 14195 Berlin, Germany. ${ }^{3}$ Small Animal Clinic, Department of Veterinary Medicine, Freie Universität Berlin, 14163 Berlin, Germany.

Received: 7 December 2017 Accepted: 30 May 2018

Published online: 08 June 2018

\section{References}

1. Helwa I, Cai J, Drewry MD, Zimmerman A, Dinkins MB, Khaled ML, et al. A comparative study of serum exosome isolation using differential ultracentrifugation and three commercial reagents. PLoS One. 2017:12(1): e0170628

2. Li M, Rai AJ, DeCastro GJ, Zeringer E, Barta T, Magdaleno S, et al. An optimized procedure for exosome isolation and analysis using serum samples: application to cancer biomarker discovery. Methods. 2015:87:2630.

3. Urbanelli L, Magini A, Buratta S, Brozzi A, Sagini K, Polchi A, et al. Signaling pathways in exosomes biogenesis, secretion and fate. Genes (Basel). 2013; 4(2):152-70

4. Zhang J, Li S, Li L, Li M, Guo C, Yao J, et al. Exosome and exosomal microRNA: trafficking, sorting, and function. Genomics Proteomics Bioinformatics. 2015;13(1):17-24.

5. Kumar D, Gupta D, Shankar S, Srivastava RK. Biomolecular characterization of exosomes released from cancer stem cells: possible implications for biomarker and treatment of cancer. Oncotarget. 2015;6(5):3280-91.

6. Mathivanan S, Ji H, Simpson RJ. Exosomes: extracellular organelles important in intercellular communication. J Proteome. 2010;73(10):1907-20.

7. Vlassov AV, Magdaleno S, Setterquist R, Conrad R. Exosomes: current knowledge of their composition, biological functions, and diagnostic and therapeutic potentials. Biochim Biophys Acta. 2012:1820(7):940-8.

8. Simons M, Raposo G. Exosomes-vesicular carriers for intercellular communication. Curr Opin Cell Biol. 2009:21(4):575-81.

9. Simpson RJ, Lim JW, Moritz RL, Mathivanan S. Exosomes: proteomic insights and diagnostic potential. Expert Rev Proteomics. 2009;6(3):267-83.

10. Lötvall J, Hill AF, Hochberg F, Buzás El, di Vizio D, Gardiner C, et al. Minimal experimental requirements for definition of extracellular vesicles and their functions: a position statement from the International Society for Extracellular Vesicles. J Extracell Vesicles. 2014;3:26913.

11. Tickner JA, Urquhart AJ, Stephenson S-A, Richard DJ, O'Byrne KJ. Functions and therapeutic roles of exosomes in cancer. Front Oncol. 2014;4:127.

12. DeVinney R, Gold WM. Establishment of two dog mastocytoma cell lines in continuous culture. Am J Respir Cell Mol Biol. 1990;3(5):413-20.

13. Théry C, Amigorena S, Raposo G, Clayton A. Isolation and characterization of exosomes from cell culture supernatants and biological fluids. Curr Protoc Cell Biol. 2006;Chapter 3(Unit 3):22. https://doi.org/10.1002/0471143030. cb0322s30.

14. Salomon C, Yee S, Scholz-Romero K, Kobayashi M, Vaswani K, Kvaskoff D, et al. Extravillous trophoblast cells-derived exosomes promote vascular smooth muscle cell migration. Front Pharmacol. 2014;5:175.

15. Wu Y, Deng W, Klinke DJ. Exosomes: improved methods to characterize their morphology, RNA content, and surface protein biomarkers. Analyst. 2015;140(19):6631-42.

16. Zhang $X$, Yuan $X$, Shi H, Wu L, Qian H, Xu W. Exosomes in cancer: small particle, big player. J Hematol Oncol. 2015;8:83.

17. Mørk M, Pedersen S, Botha J, Lund SM, Kristensen SR. Preanalytical, analytical, and biological variation of blood plasma submicron particle levels measured with nanoparticle tracking analysis and tunable resistive pulse sensing. Scand J Clin Lab Invest. 2016;76(5):349-60.

18. Tang $Y-T$, Huang $Y-Y$, Zheng $L$, Qin $S-H, X u X-P, A n T-X$, et al. Comparison of isolation methods of exosomes and exosomal RNA from cell culture medium and serum. Int J Mol Med. 2017;40(3):834-44.

19. Gheldof D, Hardij J, Cecchet F, Chatelain B, Dogné J, Mullier F. Thrombin generation assay and transmission electron microscopy: a useful 
combination to study tissue factor-bearing microvesicles. J Extracell Vesicles. 2013;2(1):19728.

20. Green TM, Alpaugh ML, Barsky SH, Rappa G, Lorico A. Breast cancer-derived extracellular vesicles: characterization and contribution to the metastatic phenotype. Biomed Res Int. 2015;2015:634865.

21. Singh R, Pochampally R, Watabe K, Lu Z, Mo Y. Exosome-mediated transfer of miR-10b promotes cell invasion in breast cancer. Mol Cancer. 2014;13:256.

22. Whiteside TL. Tumor-derived exosomes and their role in cancer progression. Adv Clin Chem. 2016;74:103-41.

23. King HW, Michael MZ, Gleadle JM. Hypoxic enhancement of exosome release by breast cancer cells. BMC Cancer. 2012;12:421.

24. Shedden K, Xie XT, Chandaroy P, Chang YT, Rosania GR. Expulsion of small molecules in vesicles shed by cancer cells: association with gene expression and chemosensitivity profiles. Cancer Res. 2003;63(15):4331-7.

25. Zhang HG, Grizzle WE. Exosomes: a novel pathway of local and distant intercellular communication that facilitates the growth and metastasis of neoplastic lesions. Am J Pathol. 2014;184(1):28-41.

26. van der Pol E, Hoekstra AG, Sturk A, Otto C, van Leeuwen TG, Nieuwland R. Optical and non-optical methods for detection and characterization of microparticles and exosomes. J Thromb Haemost. 2010;8(12):2596-607.

27. van der Pol E, Coumans FA, Grootemaat AE, Gardiner C, Sargent IL, Harrison $P$, et al. Particle size distribution of exosomes and microvesicles determined by transmission electron microscopy, flow cytometry, nanoparticle tracking analysis, and resistive pulse sensing. J Thromb Haemost. 2014;12(7):1182-92.

28. Yang VK, Loughran KA, Meola DM, Juhr CM, Thane KE, Davis AM, et al. Circulating exosome microRNA associated with heart failure secondary to myxomatous mitral valve disease in a naturally occurring canine model. J Extracell Vesicles. 2017:6(1):1350088.

29. Ichii O, Ohta H, Horino T, Nakamura T, Hosotani M, Mizoguchi T, et al. Urinary exosome-derived microRNAs reflecting the changes of renal function and histopathology in dogs. Sci Rep. 2017;7:40340.

30. Raposo G, Stoorvogel W. Extracellular vesicles: exosomes, microvesicles, and friends. J Cell Biol. 2013;200(4):373-83.

31. Viens A, Harper F, Pichard E, Comisso M, Pierron G, Ogryzko V. Use of protein biotinylation in vivo for immunoelectron microscopic localization of a specific protein isoform. J Histochem Cytochem. 2008;56(10):911-9.

Ready to submit your research? Choose BMC and benefit from:

- fast, convenient online submission

- thorough peer review by experienced researchers in your field

- rapid publication on acceptance

- support for research data, including large and complex data types

- gold Open Access which fosters wider collaboration and increased citations

- maximum visibility for your research: over $100 \mathrm{M}$ website views per year

At BMC, research is always in progress.

Learn more biomedcentral.com/submissions 